Therefore

$$
\begin{aligned}
\text { change of energy } & =\frac{1}{2} \cdot \frac{m v^{2}}{r^{2}}\left(\mathrm{MP}^{2}-\mathrm{NQ}^{2}\right) \\
& =\frac{1}{2} \cdot \frac{m v^{2}}{r^{2}}\left(\mathrm{ON}^{2}-\mathrm{OM}^{2}\right) \\
& =\frac{1}{2} \cdot \frac{m v^{2}}{r^{2}} \cdot \mathrm{MN}(\mathrm{ON}+\mathrm{OM}) .
\end{aligned}
$$

Therefore

average force in the interval $=$ change of energy $\div \mathrm{MN}$

$$
=\frac{1}{2} \cdot \frac{m v^{2}}{r^{2}}(\mathrm{ON}+\mathrm{OM}) \text {. }
$$

Therefore in the limit,

$$
x \text {-component of force at } \mathrm{P}=\frac{m v^{2}}{r^{2}} . \mathrm{OM} \text {. }
$$

Similarly,

$$
y \text {-component of force at } \mathrm{P}=\frac{m v^{2}}{r^{2}} . \mathrm{MP} \text {. }
$$

Since these are proportional to $\mathrm{OM}$ and $M P$, the resultant force acts along the radius, and its value is

$$
\frac{m v^{2}}{r^{2}} \cdot \sqrt{\mathrm{OM}^{2}+\mathrm{MP}^{2}} \text { or } \frac{m v^{2}}{r} \text {. }
$$

Indeed it is obvious from the beginning that the force is always at right angles to the direction of motion; for the energy of the motion is constant.

W. ANderson.

Deduction of Propositions on Tangents from Propositions on Secants.-In Vol. XXII., p. 64, of the Proceedings of the Society, Professor Gibson called attention to difficulties in the treatment of tangents by the method of limits. The aim of the following remarks is to show how young students may become accustomed to regard a tangent as the limit of a secant, and at the same time to furnish a type of exercise which might encourage initiative and research in the early study of Geometry.

The relation of tangent to secant in the geometry of the circle has a well known illustration in the deduction of Euc. III., 32, from Euc. III., 21. If, in Fig. 1, DB be produced to $\mathrm{E}$, BE will 
act as a pointer while we consider the behaviour of the angle $A D B$ as $D$ steadily approaches and finally reaches $B$. The movement of the pointer helps the beginner to see what we are driving at when we say that DBE "becomes" the tangent BT when $D$ coincides with $B$, and on what ground we assert that angle ABT equals angle ACB. But a variety of illustration is required and each new result obtained needs to be proved independently, before all doubt as to the legitimacy of the process is set at rest. In the following examples the results are to be obtained after the manner of deriving Euc. III., 32, from Euc. III., 21 or 22; but they are to be regarded as suggested in this way, and are to be verified afterwards by independent proof-usually, indeed, by using a proposition on tangents instead of a proposition on chords or secants. Of course the method is to be taught as sound doctrine laid down by mathematicians for the acceptance of students; the history of the doctrine is truly followed in showing that it is based on a large number and variety of facts.

\section{First Example.}

(a) If two circles intersect at $P$ and $Q$ (Fig. 2), and if lines $A P C$ and BQD be drawn meeting one circle in $A$ and $B$ and the other in $C$ and $D$, then $A B$ is parallel to $C D$.

When the common chord $P Q$ is drawn, the proof follows easily.

(b) If $B$ coincides with $A$, the chord $A B$ becomes the tangent at $A$, and so, in Fig. 3, the tangent at $A$ is parallel to $C D$.

The join of $P$ and $Q$ remains as a construction. (AB may be produced so that the produced part acts as a pointer.)

(c) If $P$ coincides with $Q$, as in Fig. $4, A B$ is parallel to $C D$.

The common chord becomes the common tangent, so that we have the following construction : at $P$ draw the common tangent.

(d) If $\mathbf{P}$ and $Q$ coincide, as in Fig. 5, $A B$ is parallel to $C D$. Again for construction draw the common tangent.

(e) If $A$ and $B$ coincide and also $P$ and $Q$ coincide, then $C$ and $D$ coincide, and we have either Fig. 6 or Fig. 7 . The tangents at $A$ and $\mathbf{C}$ are parallel.

The common tangent is again a construction line.

(f) If $\mathrm{C}$ and $\mathrm{P}$ coincide and also $\mathrm{B}$ and $\mathrm{Q}$ coincide, we have Fig. 8 and $A B$ parallel to $C D$.

The construction line is the common chord. 
(g) If $\mathrm{C}$ and $\mathbf{P}$ coincide and also $\mathrm{D}$ and $\mathrm{Q}$ coincide, we have Fig. 9 and $A B$ parallel to $C D$.

Another example may be added.

\section{Second Example.}

(a) If two circles intersect in $\mathrm{P}$ and $\mathrm{Q}$, and if a chord $\mathrm{AB}$ of one circle (Fig. 10) meets the other in $C$ and $D$, then angles $A P C$ and $\mathrm{BQD}$ are equal.

Construction: Join $\mathrm{PQ}$ and produce it to $\mathrm{R}$.

Proof: $\quad \widehat{\mathrm{APC}}=\widehat{\mathrm{PCD}}-\widehat{\mathrm{PAB}}=\widehat{\mathrm{DQQR}}-\widehat{\mathrm{BQR}}=\mathrm{BQD}$.

$(\beta)$ If $\mathrm{C}$ and $\mathrm{D}$ coincide, we have Fig. 11 , when angles $\mathrm{APC}$ and $B Q C$ are equal.

(Construction as before.)

( $\gamma$ ) If $P$ and $Q$ coincide, we have Fig. 12, when angles APC and BPD are equal.

Construction: Draw the common tangent at $\mathbf{P}$.

$(\delta)$ If $\mathrm{C}$ and $\mathrm{D}$ coincide and also $\mathrm{P}$ and $\mathrm{Q}$ coincide, then as in Fig. 13, PC bisects angle APB.

(є) If $B$ and $D$ and $Q$ coincide (Fig. 14), then the angle APC equals the angle between the tangents at $Q$ to the two circles.

(§) If $\mathrm{AB}$ produced meets the second circle in $\mathrm{C}$ and $\mathrm{D}$, and if $A P$ be produced to $A^{\prime}$ (Fig. 15), then angles $A^{\prime} P C$ and $B Q D$ are equal.

(This is also true when $\mathbf{P}$ and $\mathbf{A}^{\prime}$ coincide.)

( $\eta$ ) If $B$ and $D$ and $Q$ of Fig. 15 coincide, we have Fig. 16, and the angle $A^{\prime} P C$ is equal to the angle between the tangents at $Q$.

(This is also true when $P$ and $A^{\prime}$ coincide.)

( $\theta$ ) Fig. 17 is the case of Fig. 15 when $P$ and $Q$ coincide.

( ا) Fig. 17 becomes Fig. 18 when $\mathrm{C}$ and $\mathrm{D}$ coincide.

(к) Fig. 18 becomes Fig. 19 when $\mathbf{A}$ and $\mathbf{B}$ coincide, and then angle APC is a right angle. 
DEDUCTION OF PROPOSITIONS ON TANGENTS, ETC.
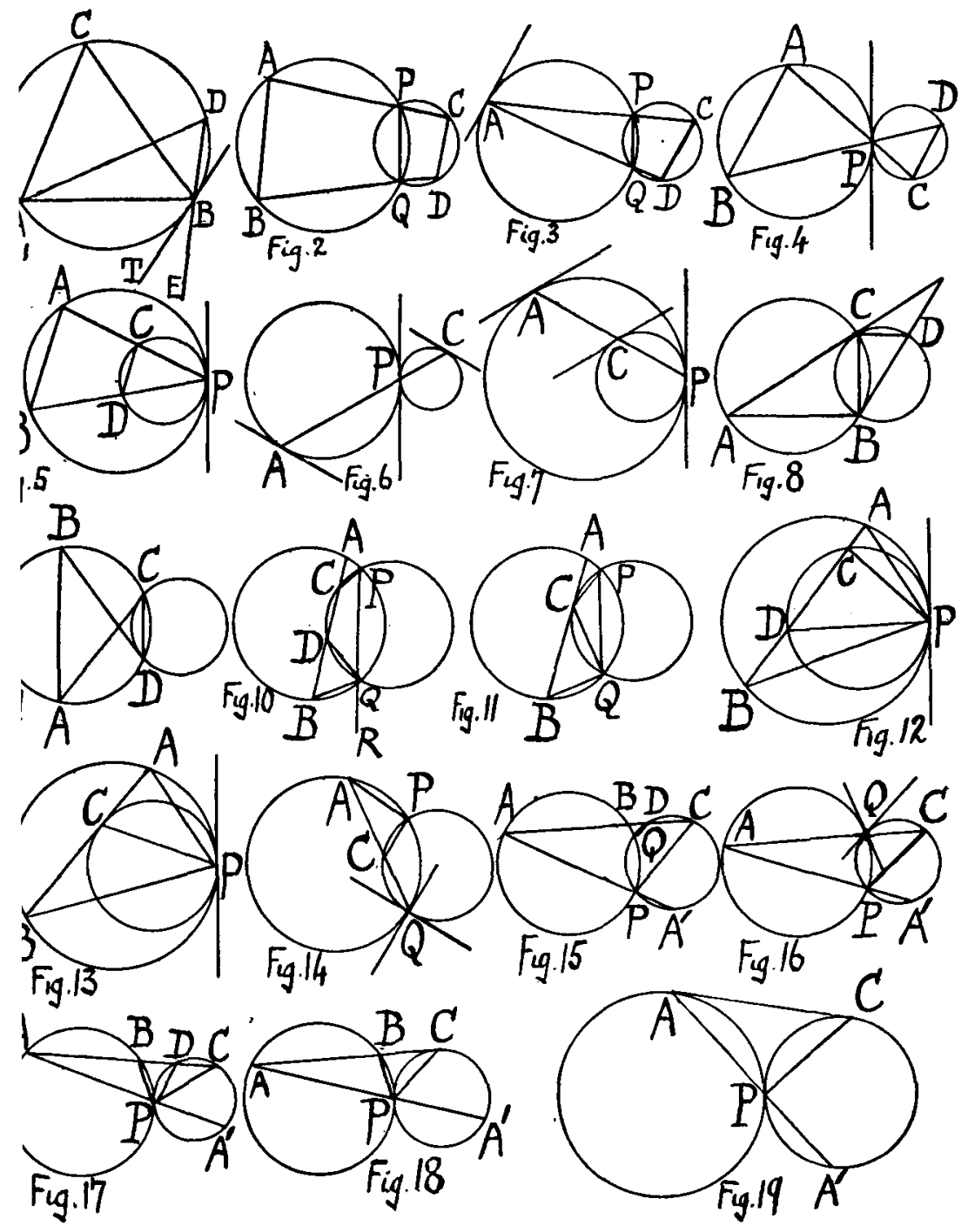

P. Pinkerton.

( 167 ) 